\title{
Maximizing geographical efficiency: An analysis of the configuration of Colorado's trauma system
}

\author{
Short Title: Optimizing trauma system configurations
}

Jan O Jansen, PhD FRCS FFICM, Division of Acute Care Surgery, Department of Surgery, University of Alabama at Birmingham, Birmingham, Alabama, USA \& Health Services

Research Unit, University of Aberdeen, Aberdeen, UK; jjansen@uabmc.edu

Ernest E Moore, MD FACS, Department of Surgery, University of Colorado, Denver \& Department of Surgery, Denver Health Medical Center, Denver, Colorado, USA;

Ernest.Moore@dhha.org

Handing Wang, PhD, Department of Computing, University of Surrey, Guildford, UK;

handing.wang@surrey.ac.uk

Jonathan J Morrison, PhD FRCS, Department of Surgery, R Adams Cowley Shock Trauma

Center, Baltimore, Maryland, USA; jimorrison@outlook.com

James D Hutchison, PhD FRCS, Department of Surgery, Medical School, University of Aberdeen, Aberdeen, UK; j.d.hutchison@abdn.ac.uk

Marion K Campbell, PhD, Health Services Research Unit, University of Aberdeen, Aberdeen, UK; m.k.campbell@abdn.ac.uk

Angela Sauaia, MD PhD, Schools of Public Health and Medicine, University of Colorado, Denver, Colorado, USA; Angela.Sauaia@ucdenver.edu 
Correspondence to: Jan Jansen, Division of Acute Care Surgery, Department of Surgery, University of Alabama at Birmingham, 1720 2nd Avenue South, Birmingham, Alabama 35294-0016

Email: $\quad$ jan.jansen@abdn.ac.uk

Phone: $\quad$ (205) 975-3030

Conflicts of Interest: The authors declare no conflicts of interest.

Source of Funding: This project did not receive specific funding.

Disclosure:

The Health Services Research Unit at the University of Aberdeen, UK, receives funding from the Chief Scientist Office of the Scottish Government Health and Social Care Directorates. The opinions expressed in this article are those of the authors alone. 


\begin{abstract}
Background: Trauma center designation in excess of need risks dilution of experience, reduction in research and training opportunities, and increased costs. The objective of this study was to evaluate the use of a novel data-driven approach (whole-system mathematical modelling of patient flow) to compare the configuration of an existing trauma system with a mathematically optimized design, using the State of Colorado as a case study.
\end{abstract}

Methods: Geographical network analysis and multi-objective optimization. 105,448 patients injured in the State of Colorado between 2009 and 2013, who met the criteria for inclusion in the state mandated trauma registry maintained by the Colorado Department of Public Health \& Environment were included. We used the Non-dominant Sorting Genetic Algorithm II (NSGAII) to conduct a multi-objective optimization of possible trauma system configurations, with the objectives of minimizing total system access time, and the number of casualties who could not reach the desired level of care.

Results: Modelling suggested that system configurations with high volume level I trauma centers could be mathematically optimized with two centers rather than the current three (with an estimated annual volume of 970-1,020 and 715-722 severely injured patients per year), 4-5 level II centers, and 12-13 level III centers. Configurations with moderate volume level I centers could be optimized with three such centers (with estimated institutional volumes of 439-502, 699-947, and 520-726 severely injured patients per year), 2-5 level II centers, and 8-10 level III centers.

Conclusions: The modelling suggested that the configuration of Colorado's trauma system could be mathematically optimized with fewer trauma centers than currently designated. Consideration should be given to the role of optimization modelling to inform decisions about the ongoing 
efficiency of trauma systems. However, modelling on its own cannot guarantee improved patient outcome; thus the use of model results for decision-making should take into account wider contextual information.

Level of Evidence: Level IV, epidemiological

Study type: Geospatial analysis

Key Words: Trauma systems, geospatial analysis, multi-objective optimization 


\section{BACKGROUND}

Injury is the principal cause of death and disability in young adults. ${ }^{1,2}$ Specialist care, provided by trauma centers, has been shown to reduce mortality and improve functional outcomes. ${ }^{3-5}$ The configuration of such a system - in terms of the number, level, and location of hospitals - is critical to balancing accessibility and institutional expertise. ${ }^{6}$ It is recommended that the majority of patients should be able to reach a level I or II trauma centers within reasonable time, which in most systems is set at 45 to 60 minutes. ${ }^{7}$ However, such centers also require a sufficient case volume to justify a specialist staffing model. ${ }^{8}$ The importance of configuring trauma systems according to need has been recognized since the $1980 \mathrm{~s},{ }^{6,7,9}$ and recently been reiterated in the National Academies' report, ${ }^{10}$ which calls for the "establishment of the appropriate number, level, and location of trauma care centers within a region, based on the needs of the population".

Most trauma systems have evolved over time rather than having been designed $a b$ initio, and few designating authorities have processes in place to guide system-wide planning. ${ }^{11}$ Economic incentives have led to proliferation and duplication of services. Between 2009 and 2012, more than 200 new trauma centers opened in the United States. ${ }^{12}$ This trend has prompted concerns about dilution of experience, reduction in research and training opportunities, and increased costs. ${ }^{11,13,14}$ The American College of Surgeons has stated that the proliferation of trauma centers could undermine the quality of care. ${ }^{16}$ A recent study of the Florida trauma system has confirmed that the designation of new trauma centers, in a mature system, was associated with a change in demographics and economics, but not performance. ${ }^{11}$ Greater recognition of these issues, and the importance of integrated care systems, ${ }^{15,17}$ has stimulated interest in the geographical configuration of trauma networks to maximize the configuration of networks according to need. ${ }^{9,11,17}$ 
The "Geospatial Evaluation of Systems of Trauma Care" (GEOS) method has established a scientific basis for configuring trauma systems and has recently been used to provide data on the development of a national trauma system in Scotland..$^{18,19,20}$ GEOS employs whole-system mathematical modelling of patient flow, using prospectively collected data, to derive the most efficient and geospatially optimized system configurations maximizing the efficient allocation of patients to the required level of care. Although originally designed to facilitate the de novo development of trauma networks, such data-driven methods could also be used to analyze the efficiency of existing systems, to explore opportunities for improvement in service efficiency. However, this approach has not been tested.

The aim of this study was to evaluate such an analysis, using routinely collected trauma registry data, to compare the configuration of an existing trauma system with a mathematically optimized design, using the State of Colorado as a case study. 


\section{METHODS}

\section{Study design}

We conducted a network analysis and multi-objective optimization. The primary outcome was the comparison of a mathematically optimized configuration of the trauma system with the current configuration - in terms of the number, level, and geographic distribution of trauma centers. The study did not attempt to quantify what impact such a reorganisation would have on clinical outcomes. The study was approved by the Colorado Multi-institutional Review Board.

\section{Sources of data}

We used data from the state mandated trauma registry maintained by the Colorado Department of Public Health \& Environment. Data were collected by individual hospitals, and then submitted to the state registry. We included patients aged 15 years and over, injured in Colorado or one of the neighboring states, and subsequently admitted or transferred to a level I, II, or III trauma center in Colorado, in 2009-2013.

\section{Setting}

The State of Colorado covers an area of $269,837 \mathrm{~km}^{2}$, with a population of 5.3 million. ${ }^{21}$ Large parts of the state are remote and rural. In 2013, there were 72 state-designated trauma centers: three level I trauma centers, all in the Denver Metro area (subsequently referred to as centers A, B, and C); nine level II trauma centers; and 21 level III trauma centers, as shown in figure 1. The remaining 39 centers were level IV and V facilities. 


\section{Data}

Overall 105,448 patients met the inclusion criteria for the study. 16,092 (15.2\%) patients did not have their incident locations recorded - for these patients we assumed that the zip code of injury was the centroid of the zip code of the admission hospital. This form of imputation was adopted to ensure the mathematical model would be conservative in its assumptions (ie would assume the model as currently configured was most appropriate).

\section{Notional prehospital triage and tasking}

Prehospital triage (using triage and tasking protocols) is widely used nationally and internationally to allocate patients to the most appropriate level of destination healthcare facility dependent on clinical need. ${ }^{22}$ Triage tools use available data (eg incident location, physiological measures etc) to inform the tasking decisions (which are protocolized decisions based on matching severity with best care). The Colorado trauma registry routinely records the data which could be used in these triage decisions. We, therefore, used the data held in the registry to 'notionally' triage the patients to the appropriate level of care according to their injury severity and clinical need (using the widely used American College of Surgeons/Centers for Disease Control algorithm. ${ }^{22}$ (Supplementary Figure 1). The algorithms are shown, and described in detail, in supplementary file 1 . Respiratory rate was recorded in 58,353 patients; systolic blood pressure in 74,750; and Glasgow Coma Scale in 44,465. Patients who met the criteria of step 1 (physiological abnormalities) or step 2 (critical injuries) of the field triage decision scheme were assigned to level I trauma center care. Patients meeting step 3 criteria (mechanism of injury) were assigned to level II trauma center, and those meeting step 4 criteria (special considerations) to level III center care. All others were assigned to level IV/V center care (table 1). The diagnostic performance of the notional triage, for detecting severe injury, was similar to that 
reported by a large study of triage, suggesting strong face validity for the model. ${ }^{23}$ The sensitivity, specificity, and positive and negative predictive values of triaged-to-level-I-centercare were $0.5,0.8,0.2$, and 0.9 respectively, and of triaged-to-level-I-or-II-center-care $0.6,0.7$, 0.2 , and 0.9 .

\section{Network analysis}

We calculated the drive- and flight-times from incident locations to trauma centers. Drive-times were calculated at both normal (based on values widely used in the logistics industry) and "light $\&$ siren" (10\% faster than normal) speeds, and adjusted for road type, day of week, and time of day, to account for variations in traffic conditions and traveling speeds. Where scene departure times were not recorded, we assumed that the journey took place at peak times. As incident locations were geocoded by zip codes, we used centroids as the origin. Exact geo-locations were used for all hospitals. The calculated travel times were broadly comparable to those recorded in the registry. Flight-times were calculated by combining the flight time from bases to incident locations and then to hospitals, with an additional 10 minutes of stand-to and 10 minutes of loading time.

We then modeled every mathematically possible configuration of trauma system, assuming that trauma centers' current designation level represented the highest possible level. We used the simulated tasking algorithm (supplementary file 1), to match patients' needs, as indicated by their preferred level of care, with available resources to determine which center each patient would have gone to, given a particular configuration, and an access time threshold (the maximum permissible time to reach the desired level of care) of 45 minutes. The algorithm, which is shown in detail in supplementary figure 2 , attempts to identify a trauma center which is of a level which matches the triage category, or higher, within the 45 minute access time 
threshold, by road. If no suitable center is identified, transfer times by helicopter are evaluated, to determine whether the patient could have reached the desired level of care by air. Where patients could not have reached the desired level of care, they were recorded as "exceptions". Exceptions were weighted, with patients with greater clinical needs, being given more weight if they had to be taken to a lower level facility. (Supplementary Figure 3)

\section{Assumptions}

We assumed that helicopters had nightflying capability, and were not restricted by geographical features, such as high mountains. We also presumed that patients who were injured in the adjacent areas of neighbouring states, and who had been taken to a Colorado trauma center, were still taken to a Colorado center. Based on a registry analysis of current transfer rates, we furthermore assumed that $36 \%$ of patients with severe injury, as defined by an ISS $>15$, who were not primarily taken to a level I or II trauma center were transferred secondarily, to the nearest level I trauma center. The triage model did not make allowance for provider judgement.

\section{Selection of optimized configurations}

We used the Non-dominant Sorting Genetic Algorithm II (NSGA-II) to conduct a multiobjective optimization of the modeled solutions, with the dual objectives of (a) minimizing total system access time, and (b) minimizing the number of weighted exceptions. We also applied a number of constraints. In particular, we specified a minimum case volume threshold of at 400 (moderate volume) and 650 severely injured patients per year (high volume), reflecting current evidence, ${ }^{24-26}$ and a maximum of seven flights per helicopter, per day, reflecting approximate mission durations. The methodology has been described in detail in a previous paper. ${ }^{27}$ In brief, for every mathematically possible configuration of trauma system, the total travel time $f_{1}$, of all 
patients, is described by the following equation, where $T_{i}$ is the travel time of patient $i$ and $N$ is the number of patients:

$$
f_{1}=\sum_{i=1}^{N} T_{i}
$$

The second objective (the number of weighted exceptions, $f_{2}$ ) is described by another equation, where, where $L_{i}$ records whether patient $i$ is an exception, and its weighting $W_{i}$ :

$$
f_{2}=\sum_{i=1}^{N} L_{i} W_{i}
$$

Optimal systems were thus those which minimized the dual objectives of $f_{1}$ and $f_{2}$ subject to the constraints outlined above - for fuller details of the methodology see Wang et al. ${ }^{27}$ The output of NSGA-II comprises a set of optimal solutions, defined as all solutions which are not worse than any other feasible solution, considering all objectives. Data were collated using Microsoft Excel (Microsoft, Redmond, WA). The modeling and multiobjective optimization were performed using MatLab (MathWorks, Natick, MA). 


\section{RESULTS}

\section{Description of population}

Overall 105,448 patients met the inclusion criteria (table 1). 13,932 (13.2\%) were severely injured (ISS>15), 13,831 (13.1\%) had suffered moderate injuries (ISS 9-15), and 77,685 (73.7\%) minor injuries (ISS 1-8). 2,833 patients (2.7\%) were retrieved by helicopter ("scene flights"), of which $43.5 \%$ were severely injured. 3,171 (3.0\%) underwent inter-facility aeromedical transfer, of which $47.7 \%$ were severely injured. In total, 18,175 patients $(20.8 \%)$ were transferred to a level I, II, or III center, from another facility. $19.8 \%$ of these were severely injured. The incidents' geographical distribution is shown in figure 1.

\section{System configurations based on high volume level I centers}

There were ten optimized configurations for a trauma system with high-volume level I trauma centers. The results are summarised in table 2 (first column), and shown in detail in figure 2 (upper part). Each row represents a trauma system configuration. The columns provide a summary of the system configuration (in terms of the number of level I, II, III and IV/V centers, shown in black), and indicate the optimized designation level of each center, within each configuration. Centers which are designated as level I centers are shown in red, and those designated as level II and III in blue and green, respectively. These hypothetical designations are contrasted with the centers' current designations, shown in the first row, and colour-coded in the same way.

All of these configurations had two level I trauma centers, four or five level II trauma centers, and either twelve or thirteen level III trauma centers. Hospitals B and C featured as level I trauma centers in all ten configurations. Hospital B would have an estimated annual case volume 
of 970 to 1,020 and hospital $\mathrm{C}$ of 715 to 722 severely injured patients. Hospitals $\mathrm{G}$ and $\mathrm{J}$ featured as level II trauma centers in all ten optimized configurations and hospitals A, E, K, L and M in some. In terms of level III facilities, hospitals N, Q, R, S, W, X, Y, FF, and HH featured in all configurations and hospitals E, K, L, AA, CC, EE, and GG in several others.

The estimated median access time for patients triaged to level I center care would be 16.3 to 17.3 minutes and 18.4 to 20.5 minutes for patients triaged to level II center care. The total number of scene flights, for patients triaged to level I, II, or III care, would be 2,459 to 2,573 per year. An estimated 1,446 or 1,447 patients who had been triaged to level I, and between 272 to 368 patients triaged to level II trauma center care would not be able to reach this level of care, and would have to be taken to a lower level facility.

Figure 3 (middle panel) shows the expected case volume, per year, of severely injured patients, in all current level I-III centers, for an optimized configuration (serial 2 in figure 2) with a volume threshold of 650 patients, and compares it against actual, current case volumes (lower panel). The mathematically optimized configuration had two level I, four level II, thirteen level III, and 53 level IV/V centers. Compared with the actual configuration, the level I centers would have a greater volume of severely injured patients. The number of severely injured patients seen in level II centers would decrease, in some cases markedly.

\section{System configurations based on moderate volume level I centers}

There were eleven mathematically optimized configurations for a system with level I trauma centers with a moderate annual case volume. The results are again summarised in table 2 (first column), and shown in detail in figure 2 (lower half). All of these configurations have three level I trauma centers (hospitals A, B, C). The estimated annual case volume of these centers would be 
439-502, 699-947, and 502-726 severely injured patients, respectively. The optimized network configurations would have two to five level II trauma centers and eight to ten level III trauma centers. Hospital J featured as a level II center in all these configurations and hospitals E, F, G, H, K, L in some. Hospitals N, S, X, Y, FF and HH again appeared as level III facilities in all configurations as well as hospital BB. Hospitals K, U, V, EE, and GG feature in some of these solutions.

The estimated median access time for patients triaged to level I trauma centers would be 18.4 to 20.7 minutes and 16.2 to 20.9 minutes for patients triaged to level II centers. There would be 2,510 to 2,892 total number of scene flights per year for patients triaged to level I, II, or III An estimated 1,442 or 1,443 patients triaged to level I and 270 to 397 patients triaged to level II trauma centers would not be able to reach this level of care and would have to be taken to a lower level facility.

Figure 3 shows the expected annual case volume. The mathematically optimized configuration (serial 18 in figure 2) had three level I, five level II, ten level III, and 54 level IV/V trauma centers. Compared with the actual configuration, the level I centers would also see a greater volume severely injured patients, again largely drawn from existing level II centers. The number of severely injured patients seen in level II centers would decrease, but also be redistributed. 


\section{DISCUSSION}

The role of trauma systems in reducing the burden of injury is widely recognized, and the continued development of such systems is strongly supported by organizations such as the American College of Surgeons, ${ }^{6}$ and the World Health Organization. ${ }^{15,17}$ The relationship between time, distance, and volume is pivotal to trauma system development, implementation, and performance, and continues to excite important discussion. This current study has successfully demonstrated the feasibility of modeling patient flow through complex care systems based on routinely collected trauma registry data; we have demonstrated that registry-based patient-level data can be re-formulated into patient-level scenarios with sufficient granularity to:

(1) be able to allocate patients to a triage category, and (2) model to the most appropriate facility the patient should have gone to (if the widely accepted principles of tasking EMS resources had been adopted at source). The study also shows the potential value of the technique in reviewing the configuration of current systems of care, with a view to improving the "geographical efficiency" of the system. (A geographically efficient system seeks to ensure the majority of patients to reach the desired level of care, in the least amount of time.)

\section{Key findings and implications}

The most striking finding was the relatively small number of centers, of all levels, suggested by the mathematical optimization. The mathematically optimal configurations of a trauma system, as expected, varied with the desired minimum volume threshold of the level I trauma centers. This is a contentious issue - few doubt the existence of a volume-outcome relationship in trauma care, but the precise position of the inflection point is not known, and the threshold for optimizing research and education/training remains elusive. This study therefore modeled two different thresholds. Mathematical optimization methodology suggested that system 
configurations with high-volume level I centers could be delivered with only two such institutions in Colorado, whereas systems with a moderate minimum volume threshold would be optimized with three level I centers. The estimated case volume of these centers would be related to the minimum volume threshold, although the exact case volumes vary, as a result of the spatial distribution of the incidents.

The findings with regards to the level I trauma centers could be regarded as largely intuitive and explained by the centroidal nature of the geographical distribution of the injuries, and the proximity of the candidate hospitals. The analysis of the level II and level III centers is, in some ways, more noteworthy: The number of centers suggested by the optimized model is smaller than the number currently designated, and there is a high degree of consistency across the solutions. In contrast to the case volumes of the level I centers, other performance variables - such as access times, number of scene flights, and number of patients who could, for geographical reasons, not reach the correct level of care - show little variation, regardless of the number of level I trauma centers. However, if a system configuration with high-volume level I centers were adopted, there would be a marked reduction in the case volume of severely injured patients in level II centers, after interfacility transfers. The effect is less pronounced if a configuration with a threshold of 400 severely injured patients per year were adopted. Assuming the well validated relationship between volume and outcome holds across all levels of trauma care, such reductions in case volume could potentially impact on the outcomes of patients who are not transferred.

\section{Comparison of actual and mathematically optimized configurations}

The study succeeded in comparing different network configurations, in terms of the number and location of centers and the potential for geographical efficiency. Comparing the predicted and actual performance (and ultimately patient outcome) is, however, more difficult. Clearly one 
cannot guarantee that a more geographically efficient system will, by definition, lead to better outcomes; however, if one accepts that the well-documented relationship between access to specialist care and patient outcome holds, a geographically efficient system could be regarded as a proxy of a system configuration that would support this underlying premise.

\section{Generalizability}

Colorado, like many regions of the US, struggles with the issue of trauma center overdesignation in urban regions, and insufficient facilities in rural areas. The State of Colorado is essentially rectangular, with the Denver metro area at its centre. It thus presents a natural model for a "huband-spoke" system configuration. The generalizability of the model to populations with more variable distributions could be questioned, but the Scottish study has shown its value in such a setting. ${ }^{18,19,20}$ When hospitals were in close proximity, the selection was based purely on geographical grounds. In practice, small decreases in geospatial effectiveness may be acceptable if there are marked differences in capability or performance of adjacent centers.

Several previous studies have attempted to address the question of how to best configure a trauma system: The TRAMAH (Trauma Resource Allocation Model for Ambulances and Hospitals) model was designed to optimally locate aeromedical depots as well as trauma centers, as applied to the Maryland Trauma System. ${ }^{28}$ The model, written in Fortran, was very advanced for its time, but was also limited by the granularity of the geocoding, the use of place of residence as a surrogate for location of injury, and the use of injury severity scores, rather than triage decisions. A recent study of the Pennsylvania Trauma System used the Network Analyst function in ArcGIS, a widely used Geographical Information System software, to evaluate the siting of up to six additional trauma centers. ${ }^{29}$ This model also relied on place of residence as a surrogate for location of injury. In contrast to these studies, the GEOS methodology relies on 
actual incident location (rather than a surrogate), and triage decisions using a widely used triage protocol, which are more realistic determinants of patient flow. More recently, the American College of Surgeons Committee on Trauma has developed a Needs-Based Assessment of Trauma Systems (NBATS) tool. The tool, which has not been widely validated, combines information on population size, transport time, community support, and the number of severely injured patients discharged from hospitals that are not designated level I, II, or III trauma centers, to give a summary score, which relates to the number of centers needed in a given area. Although helpful and relatively easy to calculate, this method does not allow the comparison of the relative merit of a particular facility becoming a trauma center. ${ }^{30}$

\section{Limitations}

The Colorado State trauma registry only mandates level I, II, and III trauma centers to record data. Patients admitted to level IV and V centers are therefore not included in the registry, except when subsequently transferred to a level I, II, or III center. The case volumes for level IV and V centers thus represent underestimates, and direct comparisons of case volumes between actual and mathematically optimized configurations will be inaccurate. However, most severely injured patients admitted to these facilities are likely to be transferred, and this selection bias is therefore unlikely to impact on the results. Nevertheless, consideration should be given to broadening registry inclusion criteria, or linkage with EMS data, to gain a more accurate picture of case volume and distribution. However, such linkage is often complex, both in terms of securing the necessary permission, and the technical challenges of matching records.

The data used to inform the triage decisions were re-constituted from registry data. However, the data did include the parameters that would be used in triage decisions eg physiology etc. The use of triage decisions is key to modeling pre-hospital patient flow through trauma systems, since 
injury severity scores are not known at this stage. Despite their importance, triage decisions are often poorly recorded. The registry also contains precise information on incident locations, but this study - for confidentiality reasons - used coarser geocoding, and the use of ZIP code centroids,. (However, of all the ZIP code centroids in Colorado, all but five were within 3 miles of the nearest road node.) The resulting low geographical resolution impacts the calculation of travel times. To facilitate future research of this kind, consideration should be given to using more precisely geocoded incident location data.

This study represents a mathematical model, which, by definition relies on a number of assumptions. However, our model has explicitly been informed by actual patient data, actual incident locations; actual locations of trauma facilities and use of widely-used triage protocols all which will improve the overall fidelity of the analysis. Also, as outlined above the model has not explicitly considered impact on patient outcome (only through direct extension of the known volume-outcome relationship previously evidenced in the trauma literature). As such, the impact of any reconfigured trauma system model on outcome cannot be guaranteed. Weather and flying conditions are difficult to model, because the decision to fly is subjective, and because conditions at the origin and destination, and the intervening flight path, may differ. Similarly, fixed-wing inter-facility transfers are also difficult to model, because they include transfers to and from airports. It also assumes that all hospitals of a certain level provide the same level of care. Mathematical models cannot account for less quantifiable characteristics, such as center maturity, individual experience; and medical politics and social economics, which may influence health care policy makers' decisions regarding system configurations, or patient preferences. This particular model, furthermore, does not evaluate cost-effectiveness. Although complex, it has recently been demonstrated that such modelling is possible. ${ }^{11}$ 
Missing incident location data is also problematic - there were around $15 \%$ of cases which required location imputation in this dataset. Imputing the centroid of the receiving hospital centroid as the incident location for these patients (the method we adopted in this study) may introduce errors, but was chosen to make the results of our model conservative - ie most in line with the existing service provision. It also preserved the case volume for the system as a whole. The aim of this study was to compare the configuration of an existing trauma system with a mathematically optimized design, in terms of the number, designation, and location of its centers. The study did not attempt to predict whether a reconfiguration, along the lines suggested, would result in improved clinical outcomes. As such, the result of any model should not be considered in isolation for decision-making and must be considered in conjunction with wider contextual information. 


\section{CONCLUSIONS}

The National Academies' recent report has called for zero preventable deaths after injury, recognizing the key importance of trauma systems, and their configuration. This study has successfully demonstrated a method for the review of the geographical efficiency of existing

trauma system configurations, using routinely collected data. In our case study the modelling suggests that the State of Colorado trauma system could be geographically efficient with fewer trauma centers than the current provision. However, modelling on its own cannot guarantee improved patient outcome; thus the use of model results for decision-making should take into account wider contextual information. 


\section{AUTHOR CONTRIBUTION STATEMENT}

The study was conceived by JOJ, EEM, JJM, MKC, and AS; and designed by all authors. The

analysis was conducted by HW, JOJ, and AS. All authors contributed to the data interpretation, and the writing and revision of the manuscript. 


\section{ACKNOWLEDGEMENT}

The data used for this study were supplied by the Health Facilities and Emergency Medical Services Division of the Colorado Department of Public Health and Environment, which specifically disclaims responsibility for any analyses, interpretations, or conclusions it has not provided. 


\section{REFERENCES}

1. Rhee P, Joseph B, Pandit V, Aziz H, Vercruysse G, Kuvatunyou N, Friese RS. Increasing trauma deaths in the United States. Ann Surg. 2014;260(1):13-21.

2. World Health Organisation. The global burden of disease. 2004 update. Geneva: WHO; 2008.

3. Gabbe BJ, Simpson PM, Sutherland AM, Wolfe R, Fitzgerald MC, Judson R, Cameron PA. Improved functional outcomes for major trauma patients in a regionalized, inclusive trauma system. Ann Surg. 2012;255:1009-1015.

4. MacKenzie EJ, Rivara FP, Jurkovich GJ, Nathens AB, Frey KP, Egleston BL, Salkever DS, Scharfstein DO. A national evaluation of the effect of trauma-center care on mortality. $N$ Engl $J$ Med. 2006;354:366-78.

5. Cole E, Lecky F, West A, Smith N, Brohi K, Davenport R, and the EloTS Study Collaborators. The impact of a pan-regional inclusive trauma system on quality of care. Ann Surg. 2016;264:188-94.

6. Committee on Trauma. Resources for optimal care of the injured patient 2014. Chicago: American College of Surgeons; 2014.

7. Branas CC, MacKenzie EJ, Williams JC, Schwab CW, Teter HM, Flanigan MC, Blatt AJ, ReVelle CS. Access to trauma centers in the United States. JAMA. 2005;293:2626-33.

8. Jansen JO, Tai NRM, Midwinter J. Planning trauma care services in the UK. BMJ. 2013;346:f738. 
9. Brown JB, Rosengart MR, Billiar TR, Peitzman AB, Sperry JL. Geographic distribution of trauma centers and injury related mortality in the United States. J Trauma Acute Care Surg. $2016 ; 80: 42-9$

10. National Academies of Sciences, Engineering, and Medicine. A National Trauma Care System: Integrating Military and Civilian Trauma Systems to Achieve Zero Preventable Deaths After Injury. Washington (DC): National Academies Press (US); 2016.

11. Ciesla DJ, Pracht EE, Leitz PT, Spain DA, Staudenmayer KL, Tepas JJ. The trauma ecosystem: the impact and economics of new trauma centers on a mature statewide trauma system. J Trauma Acute Care Surg. 2017;82:1014-1022

12. Boom In Trauma Centers Can Help Save Lives, But At What Price? Kaiser Health News in collaboration with USA today. 2016. Available at: http://khn.org/news/trauma-centers/. Accessed September 1, 2016.

13. Moore EE. Change at Level I trauma centers in Colorado would impact patients. The Denver Post. 2013 Aug 16.

14. Tepas JJ, Kerwin AJ, Ra JH. Unregulated proliferation of trauma centers undermines cost efficiency of population-based injury control. J Trauma Acute Care Surg. 2014;76:576-9.

15. Hirshon JM, Risko N, Calvello EJ, Stewart de Ramirez S, Narayan M, Theodosis C, O’Neill J. Health systems and services: the role of acute care. Bull World Health Organ. 2013;91:386388.

16. American College of Surgeons Committee on Trauma. Statement on trauma center designation based upon system need. Bull Am Coll Surg. 2015;100(1): 51-52. 
17. Anderson PD, Suter RE, Mulligan T, Bodiwala G, Razzak JA, Mock C, and International Federation for Emergency Medicine (IFEM) Task Force on Access and Availability of Emergency Care. World Health Assembly Resolution 60.22 and its importance as a health care policy tool for improving emergency care access and availability globally. Ann Emerg Med. 2012;60:35-44.e3.

18. Jansen JO, Morrison JJ, Wang H, Lawrenson R, Egan G, He S, Campbell MK. Optimizing trauma system design: the GEOS (Geospatial Evaluation of Systems of Trauma Care) approach. J Trauma Acute Care Surg. 2014;76:1035-40.

19. Jansen JO, Morrison JJ, Wang H, He S, Lawrenson R, Hutchison JD, Campbell MK. Access to specialist care: Optimizing the geographic configuration of trauma systems. J Trauma Acute Care Surg. 2015;79:756-65

20. Jansen JO, Campbell MK, and (on behalf of the GEOS Investigators). The GEOS study: designing a geospatially optimised trauma system for Scotland. Surgeon. 2014;12:61-3.

21. United States Census Bureau. QuickFacts State of Colorado. Retrieved from: http://quickfacts.census.gov/qfd/states/08000.html. Accessed 7 Feb 2016.

22. Sasser SM, Hunt RC, Faul M, Sugerman D, Pearson WS, Dulski T, Wald MM, Jurkovich GJ, Newgard CD, Lerner EB, et al. Guidelines for field triage of injured patients: recommendations of the National Expert Panel on Field Triage, 2011. MMWR. 2012;61(RR-1):1-20

23. Newgard CD, Zive D, Holmes JF, Bulger EM, Staudenmeyer K, Liao M, Rea T, Hsia RY, Ewen Wang N, Fleischman R, et al. A multisite assessment of the American College of Surgeons Committee on Trauma field triage decision scheme for identifying seriously injured children and adults. J Am Coll Surg. 2011;213:709-21. 
24. Brohi K, Parr T, Coats T. Regional trauma systems. Interim guidance for commissioners. Regional trauma systems. Interim guidance for commissioners. London: RCSE; 2009.

25. National Clinical Guideline Centre. Trauma: Service delivery. London: 2015.

26. Nathens AB, Jurkovich GJ, Maier RV, Grossman DC, Mackenzie EJ, Moore M, Rivara FP. Relationship between trauma center volume and outcomes. JAMA. 2001;285:1164.

27. Wang H, Yaochu J, Jansen JO. Data-driven surrogate-assisted multi-objective evolutionary optimization of a trauma system. IEEE Transact Evol Comput. 2016; 20(6): 939-952

28. Branas CC, Mackenzie EJ, ReVelle CS. A trauma resource allocation model for ambulance and hospitals. Health Serv Res. 2000;35(2):489-507

29. Horst MA, Rogers FB, Gross BW, Cook AD, Osler TM, Bradburn EH. A novel approach to optimal placement of new trauma centers within an existing trauma system using geospatial mapping. J Trauma Acute Care Surg. 2017;83(4):705-710

30. American College of Surgeons Committee on Trauma. Needs-Based Assessment of Trauma Systems (NBATS) tool. Available from: https://www.facs.org/qualityprograms/trauma/tscp/nbats (accessed 29 October 2017) 


\section{TABLES}

Table 1: Characteristics of study population. Percentages refer to columns in each category.

\begin{tabular}{|c|c|c|c|c|c|c|c|c|}
\hline & \multicolumn{2}{|c|}{ Minor injuries } & \multicolumn{2}{|c|}{ Moderate injuries } & \multicolumn{2}{|c|}{ Severe injuries } & \multicolumn{2}{|c|}{ All patients } \\
\hline $\mathrm{n}$ & 77,685 & & 13,831 & & 13,932 & & 105,448 & \\
\hline \multicolumn{9}{|l|}{ Age (years), n (\%) } \\
\hline $15-24$ & 9,848 & $(12.7 \%)$ & 1,789 & $(12.9 \%)$ & 2,378 & $(17.1 \%)$ & 14,015 & $(13.3 \%)$ \\
\hline $25-34$ & 8,335 & $(10.7 \%)$ & 1,458 & $(10.5 \%)$ & 1,914 & $(13.7 \%)$ & 11,707 & $(11.1 \%)$ \\
\hline $35-44$ & 7,273 & $(9.4 \%)$ & 1,381 & $(10 \%)$ & 1,621 & $(11.6 \%)$ & 10,275 & $(9.7 \%)$ \\
\hline $45-54$ & 8,991 & $(11.6 \%)$ & 1,852 & $(13.4 \%)$ & 2,078 & $(14.9 \%)$ & 12,921 & $(12.3 \%)$ \\
\hline $55-64$ & 9,752 & $(12.6 \%)$ & 1,944 & $(14.1 \%)$ & 2,034 & $(14.6 \%)$ & 13,730 & $(13 \%)$ \\
\hline $65-74$ & 8,973 & $(11.6 \%)$ & 1,530 & $(11.1 \%)$ & 1,415 & $(10.2 \%)$ & 11,918 & $(11.3 \%)$ \\
\hline $75-84$ & 12,039 & $(15.5 \%)$ & 1,915 & $(13.8 \%)$ & 1,440 & $(10.3 \%)$ & 15,394 & $(14.6 \%)$ \\
\hline$>=85$ & 12,474 & $(16.1 \%)$ & 1,962 & $(14.2 \%)$ & 1,052 & $(7.6 \%)$ & 15,488 & $(14.7 \%)$ \\
\hline \multicolumn{9}{|l|}{ Gender, $\mathrm{n}(\%)$} \\
\hline Female & 38,016 & $(48.9 \%)$ & 5,321 & $(38.5 \%)$ & 4,271 & $(30.7 \%)$ & 47,608 & $(45.1 \%)$ \\
\hline Male & 39,646 & $(51.0 \%)$ & 8,506 & $(61.5 \%)$ & 9,657 & $(69.3 \%)$ & 57,809 & $(54.8 \%)$ \\
\hline Not recorded & 23 & $(0.1 \%)$ & 4 & $(0 \%)$ & 4 & $(0 \%)$ & 29 & $(0.1 \%)$ \\
\hline \multicolumn{9}{|l|}{ Mechanism of injury, n (\%) } \\
\hline Blunt & 71,933 & $(92.6 \%)$ & 13,326 & $(96.3 \%)$ & 12,603 & $(90.5 \%)$ & 97,862 & $(92.8 \%)$ \\
\hline Penetrating & 4,129 & $(5.3 \%)$ & 452 & $(3.3 \%)$ & 1,157 & $(8.3 \%)$ & 5,738 & $(5.4 \%)$ \\
\hline Unknown & 112 & $(0.1 \%)$ & 8 & $(0.1 \%)$ & 30 & $(0.2 \%)$ & 150 & $(0.1 \%)$ \\
\hline Thermal & 1,511 & $(1.9 \%)$ & 45 & $(0.3 \%)$ & 142 & $(1 \%)$ & 1,698 & $(1.6 \%)$ \\
\hline \multicolumn{9}{|l|}{ Triage category, $\mathrm{n}(\%)$} \\
\hline Level I care & 18,248 & $(23.5 \%)$ & 3,595 & $(26.0 \%)$ & 6,503 & $(46.7 \%)$ & 28,346 & $(26.9 \%)$ \\
\hline Level II care & 6,388 & $(8.2 \%)$ & 2,430 & $(17.6 \%)$ & 2,227 & $(16.0 \%)$ & 11,045 & $(10.5 \%)$ \\
\hline Level III care & 33,526 & $(43.2 \%)$ & 5,126 & $(27.1 \%)$ & 3,103 & $(22.3 \%)$ & 41,755 & $(39.6 \%)$ \\
\hline Level IV/V care & 19,523 & $(25.1 \%)$ & 2,680 & $(19.4 \%)$ & 2,099 & $(15.1 \%)$ & 24,302 & $(23.0 \%)$ \\
\hline
\end{tabular}


Table 2: Summary of optimized trauma system configurations. "Exceptions" refers to the number of patients who could not be taken to a trauma center of the level suggested by prehospital triage (or higher).

\begin{tabular}{|c|c|c|}
\hline & $\begin{array}{l}\text { High volume centers } \\
\text { (>650 SIP/year) }\end{array}$ & $\begin{array}{c}\text { Moderate volume } \\
\text { centers } \\
\text { (>400 SIP/year) }\end{array}$ \\
\hline Number of configurations & 10 & 11 \\
\hline \multicolumn{3}{|l|}{ Number of centers } \\
\hline Level I centers & 2 & 3 \\
\hline Level II centers & 4 or 5 & 2 to 5 \\
\hline Level III centers & 12 or 13 & 8 to 10 \\
\hline \multicolumn{3}{|c|}{ Case volume (severely injured patients) of level I centers (range of medians) } \\
\hline Center A & - & 439 to 502 \\
\hline Center B & 970 to 1,020 & 699 to 947 \\
\hline Center C & 715 to 722 & 520 to 726 \\
\hline \multicolumn{3}{|l|}{ Access time, minutes (range of medians) } \\
\hline Triaged to level I center care & 16.3 to 17.3 & 18.4 to 20.7 \\
\hline Triaged to level II center care & 18.4 to 20.5 & 16.2 to 20.9 \\
\hline \multicolumn{3}{|l|}{ Exceptions } \\
\hline Patients triaged to level I care & 1,446 or 1,447 & 1,442 or 1,443 \\
\hline Patients triaged to level II care & 272 to 368 & 270 to 397 \\
\hline Scene flights (patients triaged to level I-III) & 2,459 to 2,573 & 2,510 to 2,892 \\
\hline
\end{tabular}




\section{FIGURES}

Figure 1. Map of Colorado, showing the density distribution of incidents involving severe injury, by incident location ZIP code centroid, as well as the location of current level I-III trauma centers. Size of yellow circles is proportional to number of cases. Red, blue, green and grey squares indicates current level I, II, III, and IV/V trauma centers respectively. () Google Maps 2016

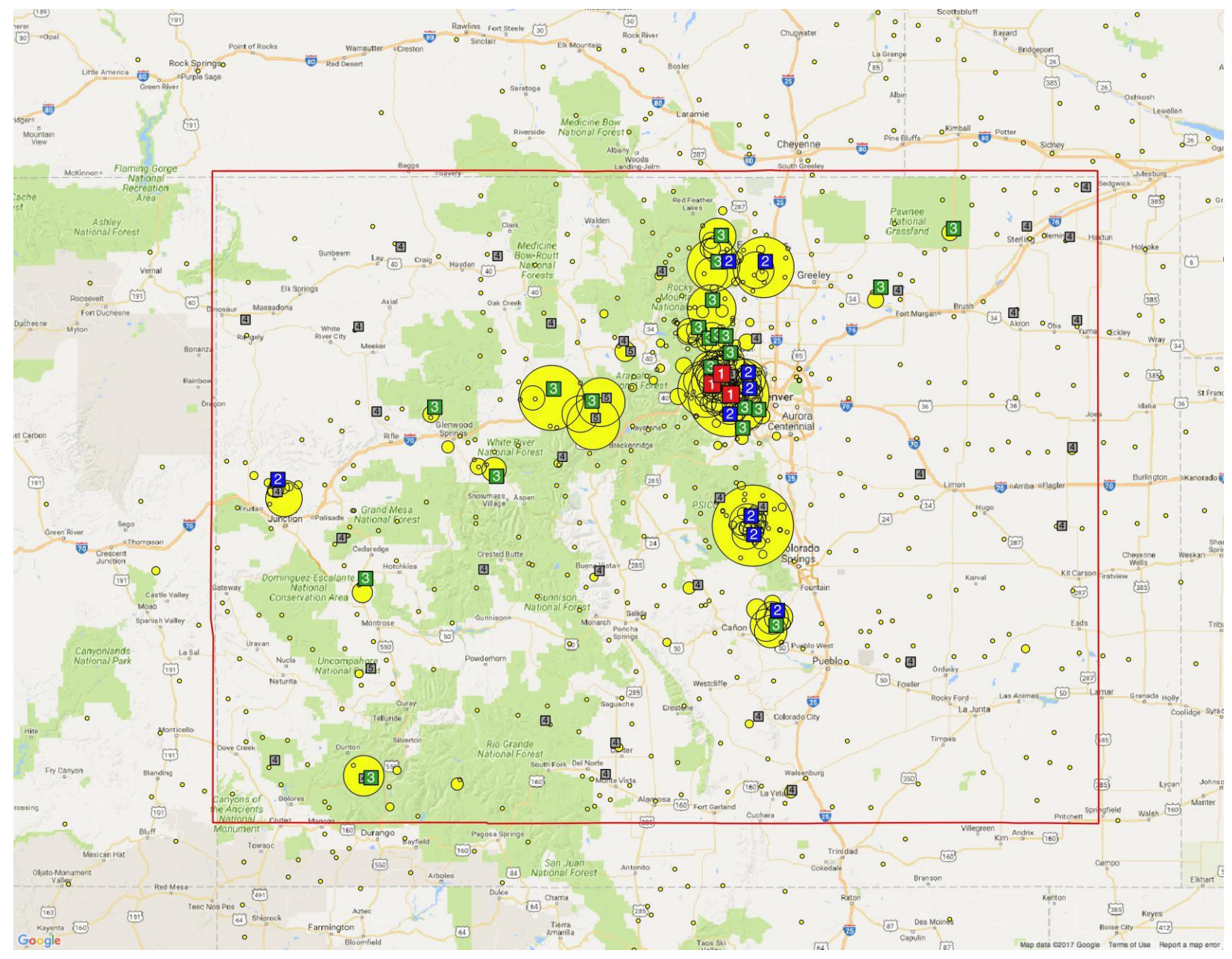


Figure 2: Optimized network configurations. Designation levels are shown in red (level I centers), blue (level II centers), and green (level III centers). Grey cells marked "L" indicate designation as a level IV center.

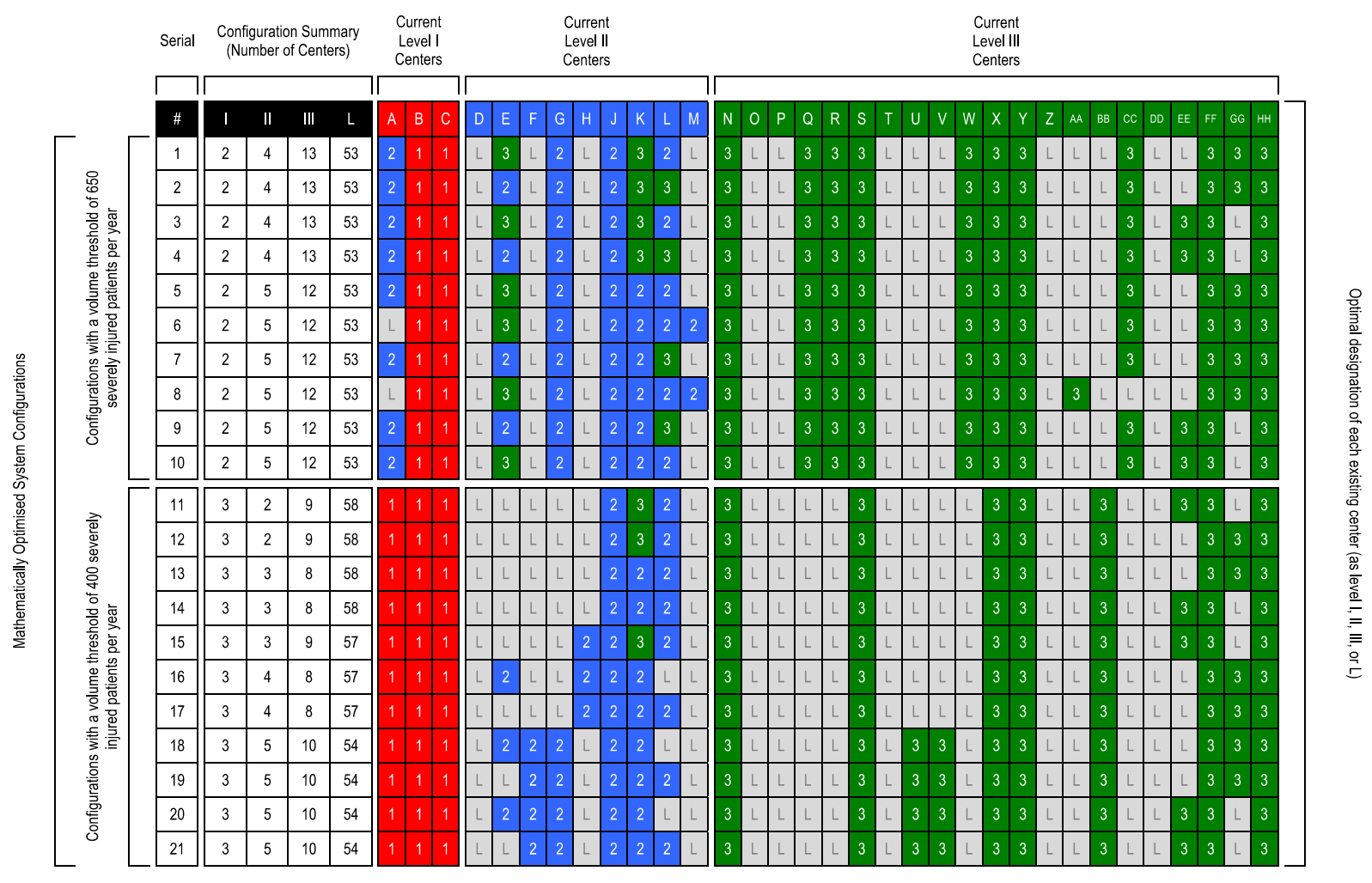


Figure 3. Distribution of severely injured patients (ISS >15), after transfers, across current level I, II and III trauma centers (bottom panel), and for two optimized configurations with volume thresholds of >400 (top panel) and >650 (middle panel) severely injured patients per year. Red, blue, and green bars indicate hospitals hypothetically designated as level I, II and III centers respectively. Grey bars indicate hospitals hypothetically designated as level IV or V centers. Figures above bars are the expected number of severely injured patients per year.

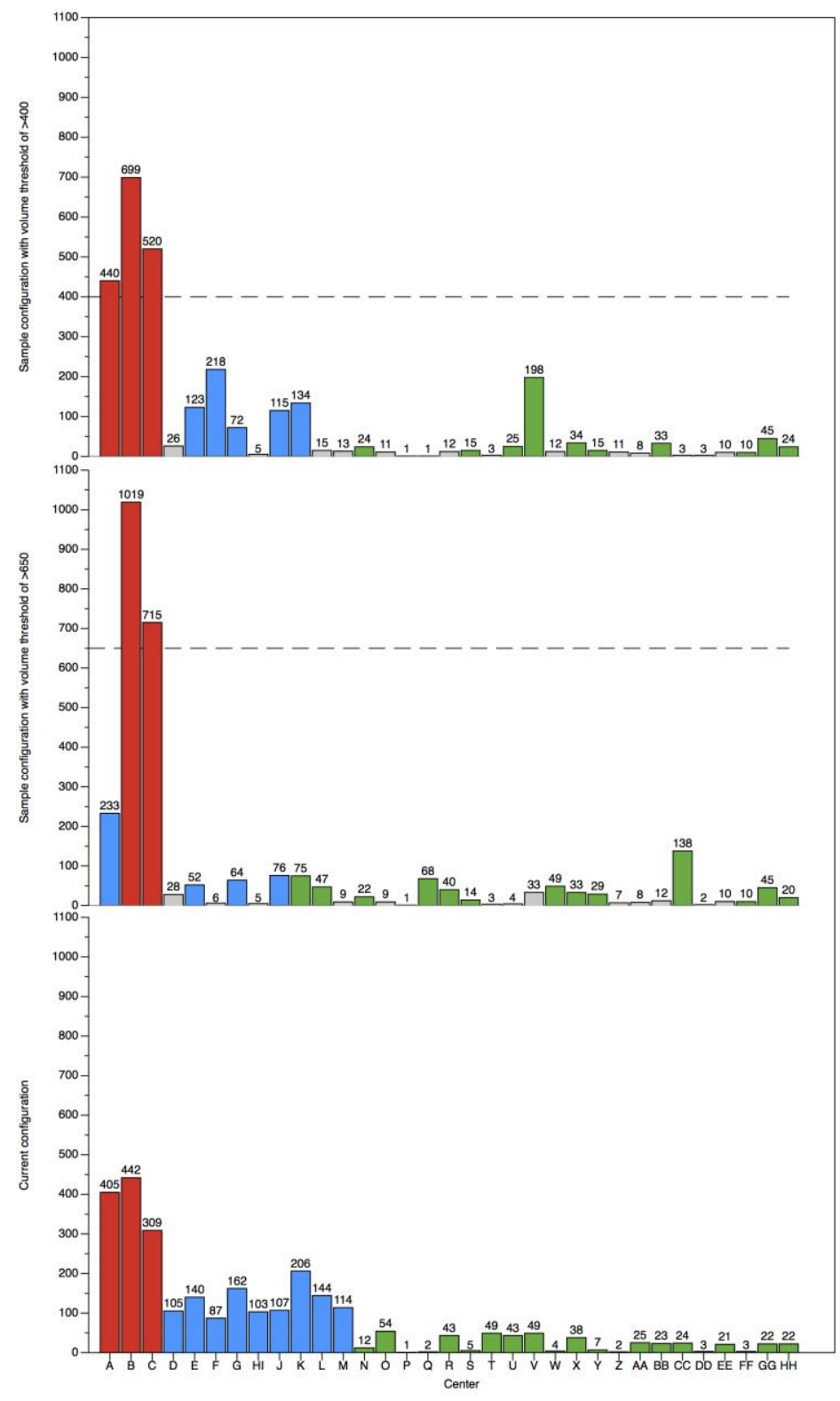

